\title{
Efficacy and onset of action of mometasone furoate/formoterol and fluticasone propionate/ salmeterol combination treatment in subjects with persistent asthma
}

David I Bernstein ${ }^{1 *}$, Jacques Hébert ${ }^{2}$, Amarjit Cheema ${ }^{3}$, Kevin R Murphy ${ }^{4}$, Ivan Chérrez-Ojeda ${ }^{5}$,

Carlos Eduardo Matiz-Bueno ${ }^{6}$, Wen-Ling Kuo ${ }^{7}$ and Hendrik Nolte ${ }^{7}$

\begin{abstract}
Background: Mometasone furoate/formoterol (MF/F) is a novel combination therapy for treatment of persistent asthma. This noninferiority trial compared the effects of MF/F and fluticasone propionate/salmeterol (FP/S) combination therapies on pulmonary function and onset of action in subjects with persistent asthma.

Methods: Following a 2- to 4-week run-in period with MF administered via a metered-dose inhaler (MDI) $200 \mu \mathrm{g}$ (delivered as 2 inhalations of MF-MDI $100 \mu \mathrm{g}$ ) twice daily (BID), subjects (aged $\geq 12 \mathrm{y}$ ) were randomized to MF/FMDI 200/10 $\mu \mathrm{g}$ BID (delivered as 2 inhalations of MF/F-MDI 100/5 $\mu \mathrm{g}$ ) or FP/S administered via a dry powder inhaler (DPI) 250/50 $\mathrm{\mu g}$ (delivered as 1 inhalation) BID for 12 weeks. The primary assessment was change from baseline to week 12 in area under the curve for forced expiratory volume in 1 second measured serially for 0-12 hours postdose ( $F E V_{1} A U C_{0-12} \mathrm{~h}$ ). Secondary assessments included onset of action (change from baseline in $\mathrm{FEV}_{1}$ at 5 minutes postdose on day 1) and patient-reported outcomes.
\end{abstract}

Results: 722 subjects were randomized to MF/F-MDI $(n=371)$ or FP/S-DPI $(n=351)$. Mean FEV $A_{1} A C_{0-12} \mathrm{~h}$ change from baseline at week 12 for MF/F-MDI and FP/S-DPI was 3.43 and $3.24 \mathrm{~L} \times \mathrm{h}$, respectively $(95 \% \mathrm{Cl},-0.40$ to 0.76$)$. MF/F-MDI was associated with a 200-mL mean increase from baseline in $\mathrm{FEV}_{1}$ at 5 minutes postdose on day 1 , which was significantly larger than the $90-\mathrm{mL}$ increase for FP/S-DPI $(P<0.001)$. The overall incidence of adverse events during the 12-week treatment period that were considered related to study therapy was similar in both groups (MF/F-MDI, 7.8\% [ $n=29] ;$ FP/S-DPI, 8.3\% [n = 29]).

Conclusions: The results of this 12-week study indicated that MF/F improves pulmonary function and asthma control similar to FP/S with a superior onset of action compared with FP/S. Both drugs were safe, improved asthma control, and demonstrated similar results for other secondary study endpoints.

Trial registration: ClinicalTrials.gov: NCT00424008

Keywords: asthma, mometasone furoate/formoterol, fluticasone propionate/salmeterol, noninferiority, onset of action

\footnotetext{
* Correspondence: bernstdd@ucmail.uc.edu

'Division of Immunology, Allergy and Rheumatology, University of Cincinnati

College of Medicine, Cincinnati, Ohio, USA

Full list of author information is available at the end of the article
} 


\section{Background}

Asthma is a chronic inflammatory disorder of the airways that results in recurrent coughing, chest tightness, wheezing, and breathlessness [1]. The first line of therapy to relieve symptoms of persistent asthma is inhaled corticosteroids (ICSs) [1,2]. However, when an ICS alone is unable to control persistent asthma, the Global Initiative for Asthma (GINA) [1] and the National Asthma Education and Prevention Program (NAEPP) [2] guidelines recommend step-up treatment with an ICS combined with a long-acting $\beta_{2}$-agonist (LABA).

The most recent ICS/LABA combination therapy indicated for the treatment of persistent asthma is mometasone furoate/formoterol delivered via metered-dose inhaler (MF/F-MDI; Dulera ${ }^{\circledR} /$ Zenhale $^{\circledR}$, Schering Corporation, a subsidiary of Merck \& Co., Inc., Whitehouse Station, NJ). Previous studies have demonstrated the safety and efficacy of MF/F-MDI 100/10 $\mu$ g twice daily (BID) [3], MF/F-MDI 200/10 $\mu \mathrm{g}$ BID [4], and MF/F-MDI $400 / 10 \mu \mathrm{g}$ BID [5] in subjects previously receiving low-, medium-, or high-dose ICS monotherapy, respectively. A long-term safety study [6] also demonstrated that MF/FMDI 200/10 and 400/10 $\mu$ g BID were well tolerated with safety profiles similar to equivalent doses of a commonly prescribed ICS/LABA combination, fluticasone propionate/salmeterol (FP/S; Advair ${ }^{\circledR} /$ Seritide $^{\circledR}$, GlaxoSmithKline, Research Triangle Park, NC). However, this 1-year safety study was not powered to compare MF/F and FP/S with regard to efficacy. The objective of the current study was to investigate, using an evaluator-blinded, noninferiority design, whether the effects of MF/F-MDI 200/10 $\mu \mathrm{g}$ BID on lung function are noninferior to those of $\mathrm{FP} / \mathrm{S}$ 250/50 $\mu \mathrm{g}$ BID (delivered via dry powder inhaler [DPI]). In addition, if noninferiority was demonstrated, the trial was designed to investigate whether the onset of action with MF/F-MDI was faster than that with FP/S-DPI.

\section{Methods}

This was a multicenter, 12 -week, ${ }^{1}$ open-label, evaluatorblinded, active-controlled, noninferiority efficacy and safety trial in subjects (aged $\geq 12$ y) with uncontrolled persistent asthma previously treated with medium-dose ICS with or without a LABA. Following a 2- to 4-week run-in treatment period with MF-MDI $200 \mu \mathrm{g}$ (delivered as 2 inhalations of MF-MDI $100 \mu \mathrm{g}$ ) BID monotherapy, eligible subjects were randomized in a 1:1 ratio according to a computer-generated randomization schedule to MF/FMDI 200/10 $\mu \mathrm{g}$ (delivered as 2 inhalations of MF/F-MDI 100/5 $\mu \mathrm{g}$ ) BID or FP/S-DPI 250/50 $\mu \mathrm{g}$ (delivered as 1 inhalation) BID for 12 weeks (Figure 1). Study visits were scheduled at screening (days -28 to -14), prebaseline (days -14 to -7 ), baseline (day 1 ), and weeks $1,2,4,8$, and 12 . The first dose of study drug was to be taken in the office under the supervision of a third-party dispenser. Written instructions on the proper use of the MDI or FP/S-DPI were provided to subjects. Subjects assigned to the MDI also used a placebo training inhaler (no DPI placebo training inhaler matching the FP/S-DPI was available for the study). The study protocol and amendments received institutional review board approval and all subjects (or subject's legal representation for those under the age of legal consent) provided written informed consent.

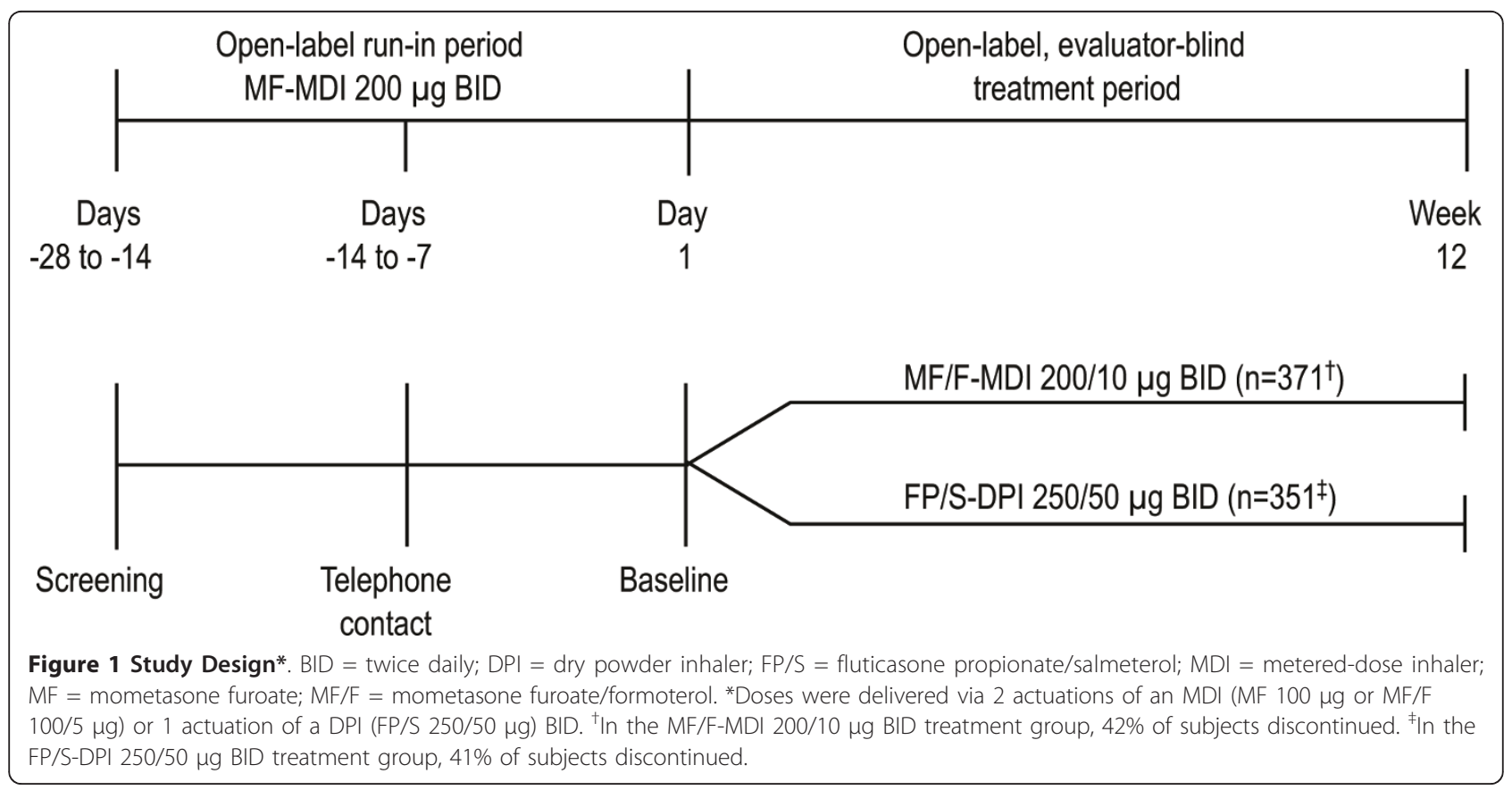




\section{Patients}

Key inclusion criteria were $\geq 12$ years of age; asthma diagnosis for $\geq 12$ months; previous treatment with a medium-dose ICS, either alone or with a LABA, for $\geq 12$ weeks before screening; stable asthma treatment regimen (daily dose unchanged) for $\geq 2$ weeks before screening; history of $\geq 2$ unscheduled asthma-related visits to a physician or emergency department within the past year, or $\geq 3$ unscheduled asthma-related visits within the past 2 years; forced expiratory volume in 1 second $\left(\mathrm{FEV}_{1}\right)$ 60\%-90\% predicted at screening and baseline; an increase in absolute $\mathrm{FEV}_{1}$ of $\geq 12 \%$ and $\geq 200 \mathrm{~mL}$ within 15-20 minutes after administration of short-acting $\beta_{2^{-}}$ agonist (SABA) rescue medication or peak expiratory flow (PEF) variability $>20 \%$; and use of $\geq 12$ inhalations of rescue medication in the final 10 days of the run-in period.

Key exclusion criteria were $>20 \%$ change in absolute $\mathrm{FEV}_{1}$ between screening and baseline; use of $>8$ inhalations per day of a SABA-MDI or $\geq 2$ nebulized treatments per day of $2.5 \mathrm{mg} \mathrm{SABA}$ on any 2 consecutive days between screening and baseline; 2 consecutive days before randomization with a decrease in PEF below the run-in stability limit, calculated over the preceding 7 days; clinical deterioration of asthma between screening and baseline that resulted in emergency treatment or hospitalization, or treatment with asthma medications other than a SABA; asthma-related emergency department visit or hospital admission in the past 3 months; current smoker or exsmoker (ie, smoked in the previous year or had a cumulative smoking history $>10$ pack-years).

\section{Assessments \\ Lung Function}

The study was designed to assess the noninferiority of MF/F-MDI 200/10 $\mu \mathrm{g}$ BID compared with FP/S-DPI 250/ $50 \mu \mathrm{g}$ BID in their effect on lung function as measured by the change from baseline (mean of 2 predose measurements on day 1) to week 12 (last observation carried forward [LOCF]) in area under the curve (AUC) in $\mathrm{FEV}_{1}$ measured serially over 0-12 hours postdose $\left(\mathrm{FEV}_{1} \mathrm{AUC}_{0-}\right.$ $12 \mathrm{~h}$ ). As a key secondary assessment, the study was also powered to assess whether MF/F-MDI 200/10 $\mu$ g BID was superior to FP/S-DPI 250/50 $\mu \mathrm{g}$ BID in onset of action (ie, change from baseline in $\mathrm{FEV}_{1}$ at 5 minutes postdose on day 1) if lung function noninferiority was demonstrated. Serial spirometry assessments of $\mathrm{FEV}_{1}$ were performed after the previous evening's dose at baseline (day 1) and the final visit (week 12) according to the following schedule: 30 minutes and immediately before the subject's morning (AM) dose of study medication; 5, 15, and 30 minutes after the AM dose; and 1, 2, 3, 4, 6, 8, 10, 11, and 12 hours after the AM dose. Additional secondary assessments included changes from baseline in trough $\mathrm{FEV}_{1}$ and AM PEF at each visit and week 12 (LOCF).

\section{Asthma Control, Quality of Life, and Symptoms}

Changes from baseline to week 12 (LOCF) in total Asthma Control Questionnaire (ACQ) [7] score and the proportion of symptom-free (ie, total asthma symptom score $=0$ [range, $0=$ none to $3=$ severe]) days and nights combined were key secondary assessments. Changes from baseline in total Asthma Quality of Life Questionnaire With Standardized Activities (AQLQ[S]) [8] score, 24-hour symptom score, and proportion of nocturnal awakenings due to asthma requiring SABA rescue medication (where baseline was the proportion of nights with nocturnal awakenings [days -7 to 1 ] before the first treatment dose) were additional secondary assessments.

\section{Clinically Judged Asthma Deteriorations}

The incidence of clinically judged asthma deteriorations, defined as asthma resulting in emergency treatment, hospitalization, or treatment with additional (excluded) asthma medication (eg, systemic glucocorticoids) were recorded throughout the study.

\section{Safety}

Safety was assessed by monitoring adverse events (AEs), clinical laboratory tests, physical examinations (including oropharyngeal examination), vital signs, and electrocardiogram (ECG) recordings throughout the study.

\section{Statistical Analyses}

Assuming a dropout rate of 5\%, a sample size of 332 subjects for each treatment group was to be enrolled to ensure that 315 subjects would be available for the test of noninferiority based on the change from baseline to week 12 (LOCF) in $\mathrm{FEV}_{1} \mathrm{AUC}_{0-12} \mathrm{~h}$ at $80 \%$ power, assuming a standard deviation of $6.7 \mathrm{~L} \times \mathrm{h}$. Noninferiority for the primary variable was achieved when the lower bound of a 2 -sided $95 \%$ confidence interval of the treatment difference (MF/F-MDI - FP/ $\mathrm{S}$-DPI) exceeded $-1.5 \mathrm{~L} \times \mathrm{h}$ in the change from baseline to week 12 (LOCF) in $\mathrm{FEV}_{1} \mathrm{AUC}_{0-12 \mathrm{~h}}$. The selected lower bound of $-1.5 \mathrm{~L} \times \mathrm{hr}$ was one-half of an estimated treatment difference of an active treatment versus placebo $(3.1 \mathrm{~L} \times \mathrm{h})$.

Changes from baseline in all assessments were analyzed by analysis of covariance, using treatment and study site as fixed effects and the corresponding baseline value for that assessment as a continuous covariate.

\section{Results}

\section{Demographics and Disposition}

A total of 722 subjects were randomized to receive MF/ F-MDI 200/10 $\mu$ g BID ( $\mathrm{n}=371$ ) or FP/S-DPI 250/50 $\mu \mathrm{g}$ BID ( $\mathrm{n}=351)$. Demographic and baseline characteristics were balanced across the 2 treatment groups. Most 
subjects were female $(459 / 722 ; 64 \%)$ and white $(624 / 722$; $86 \%$; Table 1); mean age was 44.9 years. Median duration of asthma was 12 years. Before participation in the study, most subjects were receiving 1 of the following: budesonide (monotherapy or in combination), beclomethasone (monotherapy), or fluticasone (monotherapy or in combination). Subjects had moderate persistent asthma that was uncontrolled after the MF 200- $\mu$ g BID run-in period, based on $\mathrm{FEV}_{1}$ (ie, $60 \%-80 \%$ predicted) and ACQ (ie, score $\geq 1.5$ ) findings as related to definitions from the NAEPP [2].

The percentages of subjects who discontinued the trial through week 12 were $42 \%$ and $41 \%$ in the MF/F-MDI 200/10 $\mu \mathrm{g}$ BID and FP/S-DPI 250/50 $\mu \mathrm{g}$ BID treatment groups, respectively. Administrative reasons ${ }^{2}$ were the most common cause of subject discontinuation in both treatment groups (25.6\% and $24.2 \%)$. The mean times to administrative discontinuation were 76.0 days in the MF/ F-MDI and 80.2 days in the FP/S-DPI groups, close to the 85-day treatment duration scheduled for a 12-week study. Fully $81.3 \%$ of subjects $(579 / 712)$ remained in the trial long enough to qualify for the Week 12 observed cases evaluation window, compared to the primary endpoint last-observation-carried-forward into Week 12, which includes those who discontinued earlier. Discontinuation as the result of an $\mathrm{AE}$ represented only a small proportion of subjects (approximately $2 \%$ ) in each treatment group, while discontinuation for treatment failure represented $5 \%$ of subjects in each treatment group.

\section{Lung Function}

At week 12 (LOCF), $\mathrm{FEV}_{1} \mathrm{AUC}_{0-12 \mathrm{~h}}$ for MF/F-MDI and $\mathrm{FP} / \mathrm{S}$-DPI was 3.43 vs $3.24 \mathrm{~L} \times \mathrm{h}$, respectively $(95 \% \mathrm{CI}$, -0.40 to 0.76 ), indicating that MF/F-MDI was not inferior to FP/S-DPI; noninferiority was also demonstrated on day 1 ( 3.66 vs $3.29 \mathrm{~L} \times \mathrm{h}$ [95\% CI, -0.11 to 0.84$]$ ) and week 12 (3.45 vs $3.33 \mathrm{~L} \times \mathrm{h}$ [95\% CI, -0.56 to 0.79]). Mean $\mathrm{FEV}_{1} \mathrm{AUC}_{0-12 \mathrm{~h}}$ values at week 12 (LOCF) corresponded to standardized increases from baseline of $0.29 \mathrm{~L}$ (12.7\%) for MF/F-MDI and $0.27 \mathrm{~L}$ (12.1\%) for FP/S-DPI when averaged across the 12-hour serial spirometry evaluation period (Figure 2).

Analysis of onset-of-action characteristics revealed that the effect of MF/F-MDI occurred significantly faster than the effect of FP/S-DPI (Figure 3). At 5 minutes postdose (the first scheduled measurement) on day 1 of the study, MF/F-MDI was associated with a $200-\mathrm{mL}$ least-squares (LS) mean increase from baseline in $\mathrm{FEV}_{1}$ versus a $90-\mathrm{mL}$ increase with $\mathrm{FP} / \mathrm{S}$-DPI $(P<0.001)$. When adjusting for multiplicity (using $P<0.0125$ ), the rapid, persistent effect of MF/F-MDI on $\mathrm{FEV}_{1}$ was significantly greater than the effect of FP/S-DPI for all time points measured up to 30 minutes postdose $(P<0.001$; Figure 3).

Table 1 Subject Demographics and Baseline Characteristics

\begin{tabular}{|c|c|c|}
\hline Demographic or Characteristic & $\begin{array}{c}\text { MF/F-MDI } \\
200 / 10 \mu \mathrm{g} \text { BID }(n=371)\end{array}$ & $\begin{array}{c}\text { FP/S-DPI } \\
250 / 50 \mu \mathrm{g} \mathrm{BID}(\mathrm{n}=351)\end{array}$ \\
\hline \multicolumn{3}{|l|}{ Sex, n (\%) } \\
\hline Female & $239(64)$ & $220(63)$ \\
\hline \multicolumn{3}{|l|}{ Race, n (\%) } \\
\hline White & $323(87)$ & $301(86)$ \\
\hline Mean age, y (range) & $44.8(12-82)$ & $45.1(12-80)$ \\
\hline \multicolumn{3}{|l|}{ Duration of asthma, y } \\
\hline Mean & 15.0 & 15.9 \\
\hline Median & 11.0 & 13.0 \\
\hline Prior ICS use, $\mathrm{n}(\%)^{*}$ & $225(61)$ & $188(54)$ \\
\hline \multicolumn{3}{|l|}{ Prior ICS/LABA use, $\mathrm{n}(\%)^{*}$} \\
\hline Budesonide/formoterol & $59(16)$ & $59(17)$ \\
\hline Fluticasone/salmeterol & $104(28)$ & $115(33)$ \\
\hline \multicolumn{3}{|l|}{ Mean $\mathrm{FEV}_{1}$ at baseline } \\
\hline L & 2.30 & 2.37 \\
\hline Percentage predicted & 73.8 & 74.4 \\
\hline Mean total ACQ score ${ }^{\dagger}$ & 1.80 & 1.80 \\
\hline Mean total AQLQ(S) score ${ }^{\ddagger}$ & 5.14 & 5.14 \\
\hline
\end{tabular}

$\overline{\mathrm{ACQ}}=$ Asthma Control Questionnaire; $\mathrm{AQLQ}(\mathrm{S})=$ Asthma Quality of Life Questionnaire With Standardized Activities; BID = twice daily; DPI = dry powder inhaler; $\mathrm{FEV}_{1}=$ forced expiratory volume in $1 \mathrm{~s} ; \mathrm{FP} / \mathrm{S}=$ fluticasone propionate/salmeterol; ICS = inhaled corticosteroid; LABA = long-acting $\beta_{2}$-agonist; MDI = metereddose inhaler; $M F / F=$ mometasone furoate/formoterol.

*Subjects could have received multiple types of ICS monotherapy and combination ICS/LABA therapy in the 3 months before screening.

${ }^{\dagger} A C Q$ score based on a 7-point scale that ranged from 0 (best asthma control) to 6 (worst asthma control).

${ }^{\ddagger} \mathrm{AQLQ}(\mathrm{S})$ score based on a 7-point scale that ranged from 1 (worst quality of life) to 7 (best quality of life) 


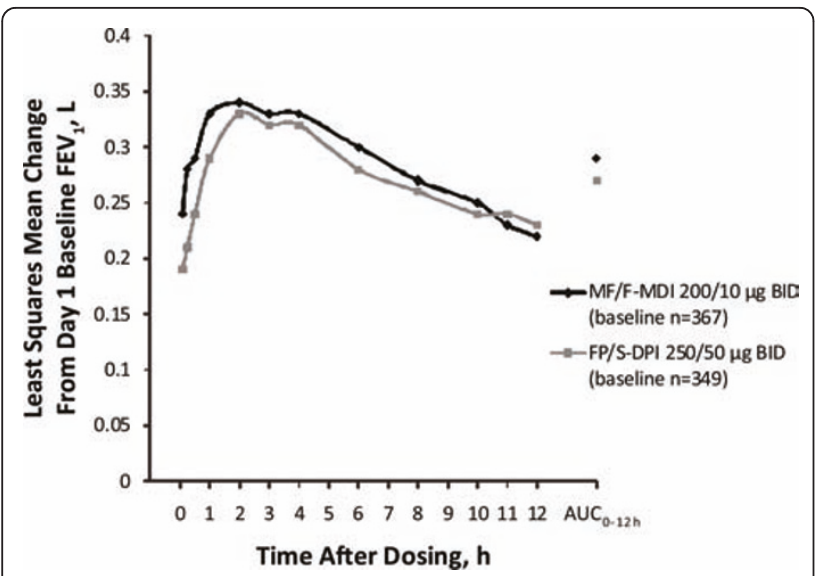

Figure 2 Serial FEV ${ }_{1}(0-12 \mathrm{~h})$ and Standardized $\mathrm{FEV}_{1} \mathrm{AUC}_{0-12 \mathrm{~h}}$ at Week 12 (LOCF). AUC = area under the curve; BID = twice daily; $\mathrm{DPI}=$ dry powder inhaler; $\mathrm{FEV}_{1}=$ forced expiratory volume in 1 second; FP/S = fluticasone propionate/salmeterol; LOCF = last observation carried forward; $\mathrm{MDI}=$ metered-dose inhaler; $\mathrm{MF} / \mathrm{F}=$ mometasone furoate/formoterol.

Baseline LS mean trough $\mathrm{FEV}_{1}$ values were 2.31 and $2.39 \mathrm{~L}$ in the MF/F-MDI and FP/S-DPI groups, respectively. Changes from baseline in trough $\mathrm{FEV}_{1}$ were not significantly different between groups at week 12 (LOCF) (0.14 and $0.17 \mathrm{~L}$, respectively) or any time during the 12-week treatment period.

Both groups had similar baseline LS mean AM PEF values (MF/F-MDI, $360.3 \mathrm{~L} / \mathrm{min}$; FP/S-DPI, $363.3 \mathrm{~L} /$ $\mathrm{min})$, and both treatments resulted in stable and positive AM PEF changes from baseline. There were no significant differences between treatments in change from

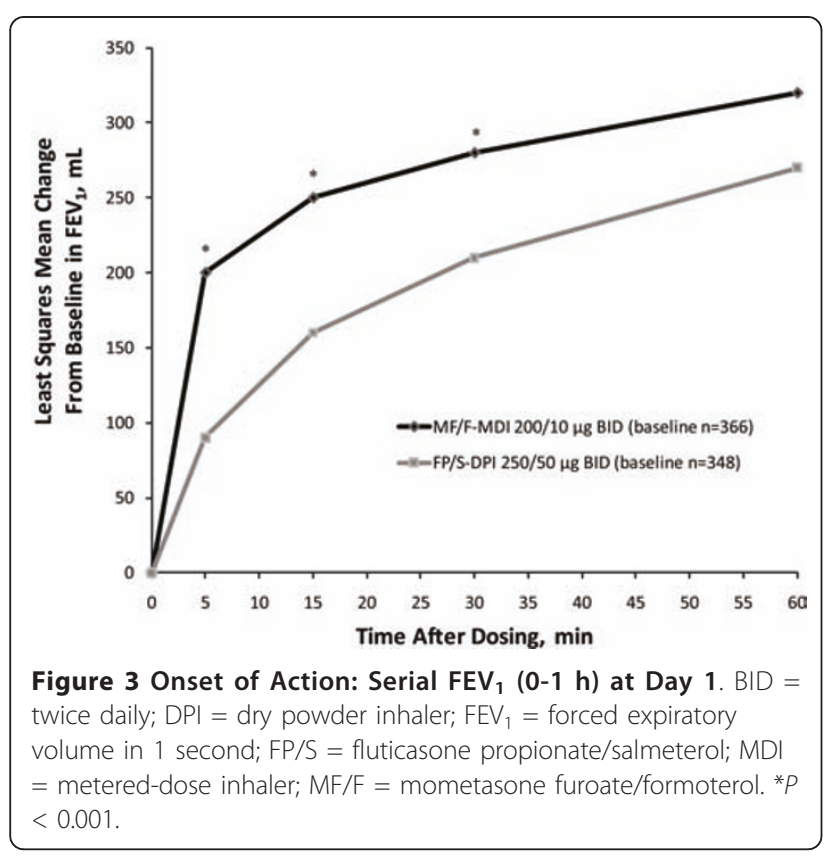

baseline LS mean AM PEF at any week or week 12 (LOCF) (MF/F-MDI, $21.3 \mathrm{~L} / \mathrm{min}$ [6.9\%]; FP/S-DPI, 23.0 $\mathrm{L} / \mathrm{min}[7.9 \%])$.

\section{Asthma Control, Quality of Life, and Symptoms}

At week 4 and week 12 (LOCF), MF/F-MDI was noninferior to FP/S-DPI in LS mean total ACQ and AQLQ(S) score changes from baseline (Figure 4). In both groups, ACQ scores improved to levels that were below the uncontrolled threshold. In addition, ACQ and AQLQ(S) score changes achieved a minimally important difference (MID; $\geq 0.5)[9,10]$ in both groups.

Both groups had the same LS mean baseline proportion of nights with nocturnal awakenings due to asthma that required the use of a SABA (0.23). Both treatments reduced this proportion by $>65 \%$ at week 12 (LOCF) (MF/F-MDI, -0.14 [-65.5\%]; FP/S-DPI, -0.16 [-69.8\%]); there was no significant difference between the groups.

Total LS mean 24-hour asthma symptom scores were similar between the groups (MF/F-MDI, 1.87; FP/S-DPI, 1.89 ). Both treatments improved (ie, reduced) LS mean symptom scores by $\geq 40 \%$ at week 12 (LOCF) (MF/FMDI, -0.82 [-40.0\%]; FP/S-DPI, -0.91 [-49.9\%]); there was no significant difference between the groups.

MF/F-MDI was found to be noninferior to FP/S-DPI in the proportion of symptom-free days and nights; both treatment groups demonstrated improvements from baseline (Figure 5).

\section{Clinically Judged Asthma Deteriorations}

The percentage of subjects who experienced a clinically judged asthma deterioration (ie, a deterioration of asthma requiring further treatment; see methods) was the same in both treatment groups (MF/F-MDI, 5.7\% [n $=21]$; FP/S-DPI, 5.7\% $[\mathrm{n}=20])$.

\section{Safety}

The incidence of AEs during the 12-week treatment period that were considered related to study therapy was similar in both groups (MF/F-MDI, 7.8\% [n = 29]; FP/SDPI, 8.3\% [ $\mathrm{n}=29])$. The most common treatment-related AEs were dysphonia (MF/F-MDI, $1.6 \%[n=6]$; FP/S-DPI, $2.8 \%$ [ $n=10]$ ), headache (MF/F-MDI, $0.8 \%$ [n = 3]; FP/SDPI, $0.9 \%[n=3]$ ), oropharyngeal pain (MF/F-MDI, $1.1 \%$ $[\mathrm{n}=4]$; FP/S-DPI, $0.6 \%[\mathrm{n}=2])$, and oropharyngeal candidiasis (MF/F-MDI, 0.5\% [n = 2]; FP/S-DPI, 0.6\% [n = 2]). Serious AEs occurred infrequently (MF/F-MDI, $1.3 \%$ $[\mathrm{n}=5]$; FP/S-DPI, $1.4 \%[\mathrm{n}=5]$ ); only 1 serious AE (ventricular extrasystoles, MF/F-MDI) was considered probably treatment related. No life-threatening AEs were reported, and no subjects died during the study. No clinically relevant changes in laboratory values, mean vital signs, or ECG measurements were observed. 


\section{Discussion}

This study in adult and adolescent subjects with persistent asthma uncontrolled on medium-dose ICS monotherapy demonstrated that treatment with MF/F-MDI 200/10 $\mu \mathrm{g}$ BID improved lung function and other patient-reported outcomes similar to FP/S-DPI 250/50 $\mu \mathrm{g}$ BID with an onset of action superior to FP/S-DPI. Although stable lung function was demonstrated during the open-label run-in period, subjects were still uncontrolled at baseline (ie, total ACQ score $\geq 1.5$ ), indicating that medium-dose MF alone was suboptimal for treating this subject population. Two key observations can be taken from the lung function results regarding the efficacy of MF/F over 12 weeks of treatment. First, treatment with MF/F-MDI 200/10 $\mu \mathrm{g}$ BID was noninferior compared with FP/S-DPI 250/50 $\mu$ g BID based on FEV 1 $\mathrm{AUC}_{0-12 \mathrm{~h}}$ at week 12 (LOCF). Second, a significant ( $\geq 200 \mathrm{~mL}$ from baseline) bronchodilator effect of the $\mathrm{MF} / \mathrm{F}$ combination was observed as early as 5 minutes postdose, which was superior to $\mathrm{FP} / \mathrm{S}$ through 30 minutes on day 1 . This result was expected given the known characteristics of these ICS/LABA combination constituents. Previous studies have demonstrated the efficacy of MF [11-14] and FP [15-17] monotherapy in improving lung function outcomes in patients with asthma. Although LABA monotherapy should not be used to treat asthma [18], formoterol and salmeterol have also been shown to be effective in improving lung function outcomes [19]. However, formoterol is associated with a faster onset of action than salmeterol $[20,21]$. As such, it does not seem surprising that $\mathrm{MF} / \mathrm{F}$ was associated with a faster onset of action than $\mathrm{FP} / \mathrm{S}$ in the current study.

As recently reviewed by Murphy and Bender [22], several surveys have indicated that rapid onset of action is a highly desirable attribute of asthma therapy from a patient perspective. The perception that controller medication is working immediately is a strong predictor of overall treatment satisfaction and may lead to improved medication adherence [22]. However, although the present study demonstrated greater improvements in pulmonary function 5-30 minutes postdose for the MF/FMDI group compared with the FP/S-DPI group, it did not measure corresponding symptom scores during that time period. Thus, the clinical relevance of the faster onset of action with MF/F-MDI compared with FP/SDPI has not been determined.

At week 12 (LOCF), a MID in total ACQ score was achieved in both treatment groups and both treatments also shifted patients to the "controlled" [23] ACQ score range. Other secondary efficacy results (eg, improvements in the AQLQ[S] total score) further indicated that MF/F-MDI 200/10 $\mu \mathrm{g}$ BID is noninferior to FP/SDPI 250/50 $\mu \mathrm{g}$ BID and that both treatments improved asthma outcomes compared with baseline measurements.

A review of the safety data indicated that both treatments were well tolerated. The number and type of AEs reported in this study were not unexpected and did not raise any new safety concerns with regard to MF/F-MDI or FP/S-DPI. The most common treatment-related AE reported was dysphonia, which is a known side effect of ICS treatment [24].

This study was originally scheduled for 1 year and was stopped early due to protracted enrollment period, which resulted in a longer than expected study duration. At the termination of the study, 12-week data were available for $81.3 \%$ of patients ( 579 of the 712 patients originally enrolled in the study), and $40 \%$ of noncompletion occurred after the termination of the study. Therefore, most of these discontinuations were a product of early trial termination. Yet because the mean times to administrative discontinuation were approximately 80 days in each treatment group-close to the 85-day treatment duration scheduled for a 12-week study-and because a large proportion of subjects (81.3\%) remained in the trial long enough to qualify for the Week 12 observed cases evaluation window, the integrity of the population for noninferiority evaluation was not compromised. Similarly, the proportions of discontinuations owing to reasons other than administrative are similar to those for other MF/F-MDI studies [3-5].

This trial was of 12 weeks' duration and, as such, does not provide insight into the longer-term efficacy and safety of MF/F-MDI. The longer-term safety of MF/FMDI has been demonstrated by Maspero et al [6]. The trial was of an open-label design and is, therefore, subject to the typical limitations inherent with open-label study. However, an open-label randomized design with frequent, uniform study visits and robust clinical endpoints, such as that used for this study, can be appropriate for comparing similar treatments to determine noninferiority. In this context, it is of interest to note the primary analysis of $\mathrm{FEV}_{1}$ AUC at endpoint favored MF/F-MDI by $20 \mathrm{~mL}$ over FP/S-DPI in a design setting that usually favors the comparator marketed product. While the study was open-label, subjects were expected to be more familiar with the treatment administration of FP/S-DPI than the use of the MF/F-MDI test product.

An important consideration with this noninferiority design was the demonstration of assay sensitivity. Thus, the subject's own treatment was discontinued at screening, and a run-in period was carried out during which subjects had to demonstrate a degree of asthma deterioration on MF monotherapy. The results show that the study population was appropriately treated with combination therapy and provided assurance that 

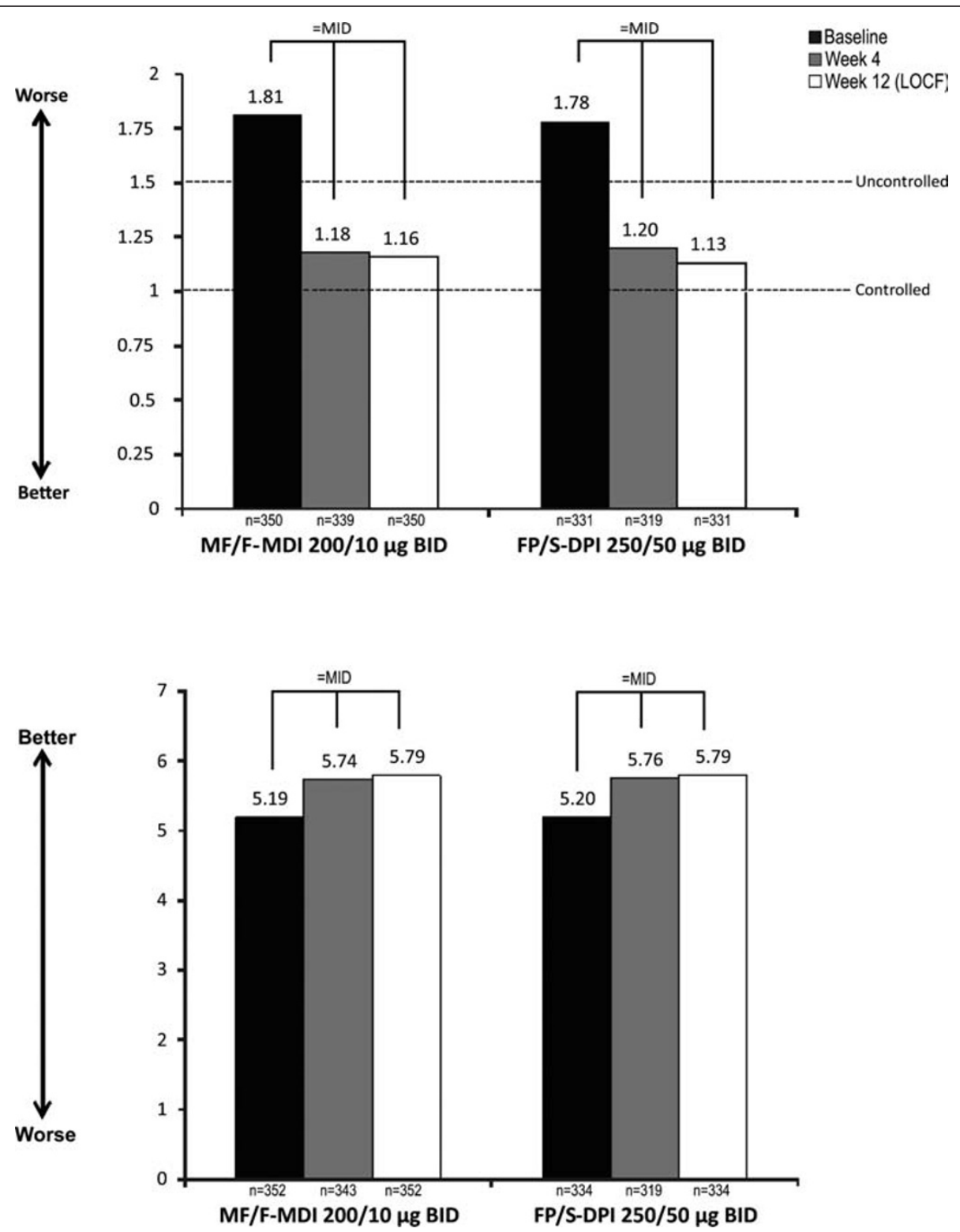

Figure 4 Mean Total ACQ* (top) and AQLQ(S) ${ }^{\dagger}$ (bottom) Scores. ACQ = Asthma Control Questionnaire; AQLQ(S) $=$ Asthma Quality of Life Questionnaire With Standardized. Activities; BID = twice daily; DPI = dry powder inhaler; FP/S = fluticasone propionate/salmeterol; $L O C F=$ last observation carried forward; $\mathrm{MDI}=$ metered-dose inhaler; $\mathrm{MF} / \mathrm{F}=$ mometasone furoate/formoterol; $\mathrm{MID}=$ minimally important difference. ${ }^{*} \mathrm{ACQ}$ score based on a 7-point scale that ranged from 0 (best asthma control) to 6 (worst asthma control). ${ }^{\dagger} A Q L Q(S)$ score based on a 7-point scale that ranged from 1 (worst quality of life) to 7 (best quality of life).

the study population was sensitive enough to detect a potential difference between the MDI and DPI formulations by demonstrating that subjects were responsive to changes in treatment. Therefore, the results indicate that subjects not well controlled on ICS monotherapy will improve lung function and asthma control to the same degree when treated with either combination. It is also important to note that the current study was adequately powered to test for superiority of the onset of action endpoint, and previous trials [3-5] have demonstrated the superiority of MF/F compared with placebo, MF, and/or formoterol for various efficacy assessments.

\section{Conclusion}

In conclusion, the results of this study indicated that MF/F-MDI 200/10 $\mu \mathrm{g}$ BID is noninferior based on $\mathrm{FEV}_{1}$ $\mathrm{AUC}_{0-12 \mathrm{~h}}$ and superior in onset of action to FP/S-DPI 250/50 $\mu \mathrm{g}$ BID over 12 weeks of treatment. Both combinations were safe, improved asthma control, and 


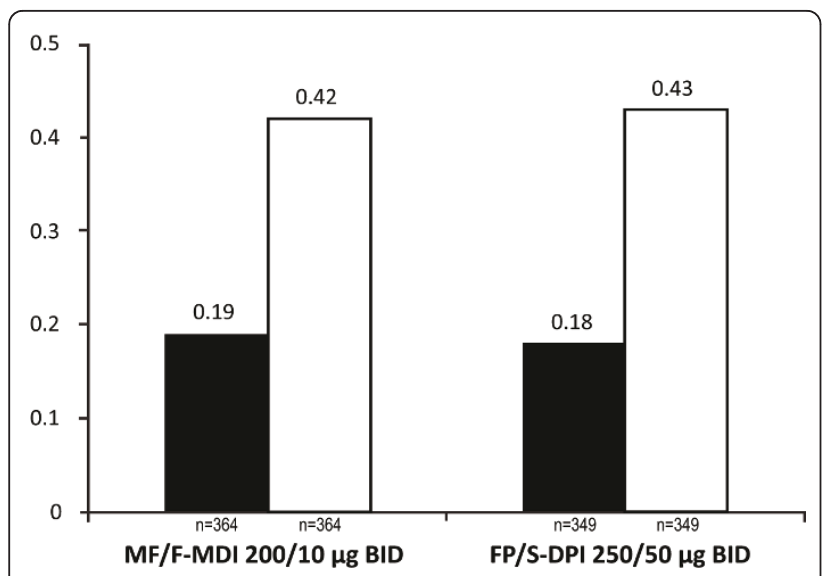

Figure 5 Proportion of Symptom-Free Days and Nights. BID = twice daily; DPI = dry powder inhaler; FP/S = fluticasone propionate/salmeterol; LOCF = last observation carried forward; MDI $=$ metered-dose inhaler; $\mathrm{MF} / \mathrm{F}=$ mometasone furoate/formoterol.

demonstrated similar results for other secondary study endpoints.

\section{Acknowledgements}

This study was funded by Merck Sharp \& Dohme Corp., a subsidiary of Merck \& Co., Inc., Whitehouse Station, NJ. Medical writing assistance and editorial support was provided by Brett D. Mahon, PhD, of Complete Publication Solutions, LLC, Horsham, PA, and Gregory Bezkorovainy, MA, of AdelphiEden Health Communications, New York, NY; this support was funded by Merck Sharp \& Dohme Corp., a subsidiary of Merck \& Co., Inc., Whitehouse Station, NJ. Additional editorial support was provided by Jorge Moreno-Cantu, PhD, Office of the Chief Medical Officer, Merck Sharp \& Dohme Corp., a subsidiary of Merck \& Co., Inc., Whitehouse Station, NJ. Endnotes

${ }^{1}$ This study was originally planned to have a second, 40-week phase extending the open-label, evaluator-blind treatment period to a total of 52 weeks to assess long-term benefits and comparative safety data. It was stopped early, however, as other blinded and placebo-controlled studies had been completed for this purpose [3-6].

${ }^{2}$ As noted above, this study had originally been planned to include a second 40-week open-label treatment period but was terminated after 12 weeks of open-label treatment. The large number of discontinuations for reasons other than adverse events or inadequate efficacy was a product of the early trial termination.

\section{Author details}

'Division of Immunology, Allergy and Rheumatology, University of Cincinnati College of Medicine, Cincinnati, Ohio, USA. ${ }^{2}$ Centre de Recherche Appliquée en Allergie de Québec, Québec, Canada. ${ }^{3}$ Alpha Medical Research, Mississauga, Ontario, Canada. ${ }^{4}$ Boys Town National Research Hospital, Boys Town, Nebraska, USA. ${ }^{5}$ RESPIRALAB Allergy, Respiratory \& Sleep Center, Guayaquil, Ecuador. ${ }^{6}$ Fundación Salud Bosque, Bogota, Colombia. ${ }^{7}$ Merck Sharp \& Dohme Corp., a subsidiary of Merck \& Co., Inc., Whitehouse Station, NJ USA.

\section{Authors' contributions}

All authors made substantial contributions to the study conception and design, or acquisition of data, or analysis and interpretation of data; have been involved in drafting the manuscript and/or revising it critically; and have given final approval of the published version.

\section{Competing interests}

David I. Bernstein has received honoraria as a member of Merck \& Co., Inc. advisory boards, served as a consultant for GlaxoSmithKline, and has been a clinical investigator for clinical trials sponsored by Merck \& Co, Inc. and GlaxoSmithKline. Jacques Hébert has received speaker/consultancy fees and research support from Merck Canada. Amarjit Cheema has received speaker fees and research support from Merck \& Co., Inc. Kevin R. Murphy has received speaker/consultancy fees and research support from AstraZeneca, Boehringer Ingelheim, Dey, Merck\& Co., Inc, Genentech, GlaxoSmithKline, and Novartis. Ivan Chérrez-Ojeda has received lecture fees from Merck \& Co., Inc. Carlos Eduardo Matiz Bueno's spouse is an employee of Merck \& Co., Inc. Wen-Ling Kuo and Hendrik Nolte are employees of Merck Sharp \& Dohme Corp., a subsidiary of Merck \& Co., Inc., Whitehouse Station, NJ.

Received: 8 July 2011 Accepted: 7 December 2011

Published: 7 December 2011

\section{References}

1. Global Initiative for Asthma: Global Strategy for Asthma Management and Prevention.[http://www.ginasthma.org/guidelines-gina-report-globalstrategy-for-asthma.html], Accessed November 15, 2011.

2. National Asthma Education and Prevention Program: Expert Panel Report 3: Guidelines for the Diagnosis and Management of Asthma. Full Report. [http://www.nhlbi.nih.gov/guidelines/asthma/asthgdln.htm], Accessed December 14, 2010.

3. Meltzer EO, Kuna P, Nolte H, S Nayak A, Laforce C, on Behalf of the P04073 Study Investigators: Mometasone Furoate/Formoterol Reduces Asthma Deteriorations and Improves Lung Function. Eur Rispirol J 2011, http://erj. ersjournals.com/content/early/2011/08/04/09031936.00020310.long. Accessed Sep 7, 2011.

4. Nathan RA, Nolte H, Pearlman DS: Twenty-six-week efficacy and safety study of mometasone furoate/formoterol 200/10 microg combination treatment in patients with persistent asthma previously receiving medium-dose inhaled corticosteroids. Allergy Asthma Proc 2010, 31(4):269-279.

5. Weinstein SF, Corren J, Murphy K, Nolte H, White M: Twelve-week efficacy and safety study of mometasone furoate/formoterol 200/10 microg and 400/10 microg combination treatments in patients with persistent asthma previously receiving high-dose inhaled corticosteroids. Allergy Asthma Proc 2010, 31(4):280-289.

6. Maspero JF, Nolte H, Cherrez-Ojeda I: Long-term safety of mometasone furoate/formoterol combination for treatment of patients with persistent asthma. J Asthma 2010, 47(10):1106-1115.

7. Juniper EF, O'Byrne PM, Guyatt GH, Ferrie PJ, King DR: Development and validation of a questionnaire to measure asthma control. Eur Respir J 1999, 14(4):902-907.

8. Juniper EF, Guyatt GH, Epstein RS, Ferrie PJ, Jaeschke R, Hiller TK: Evaluation of impairment of health related quality of life in asthma: development of a questionnaire for use in clinical trials. Thorax 1992, 47(2):76-83.

9. Juniper EF, Guyatt GH, Willan A, Griffith LE: Determining a minimal important change in a disease-specific Quality of Life Questionnaire. J Clin Epidemiol 1994, 47(1):81-87.

10. Juniper EF, Svensson K, Mork AC, Stahl E: Measurement properties and interpretation of three shortened versions of the asthma control questionnaire. Respir Med 2005, 99(5):553-558.

11. Fish JE, Karpel JP, Craig TJ, Bensch GW, Noonan M, Webb DR, Silverman B, Schenkel EJ, Rooklin AR, Ramsdell JW, Nathan R, Leflein JG, Grossman J, Graft DF, Gower RG, Garay SM, Frigas E, Degraff AC, Bronsky EA, Bernstein DI, Berger W, Shneyer L, Nolop KB, Harrison JE: Inhaled mometasone furoate reduces oral prednisone requirements while improving respiratory function and health-related quality of life in patients with severe persistent asthma. J Allergy Clin Immunol 2000, 106(5):852-860.

12. Kemp JP, Berkowitz RB, Miller SD, Murray JJ, Nolop K, Harrison JE: Mometasone furoate administered once daily is as effective as twicedaily administration for treatment of mild-to-moderate persistent asthma. J Allergy Clin Immunol 2000, 106(3):485-492.

13. Nathan RA, Nayak AS, Graft DF, Lawrence M, Picone FJ, Ahmed T, Wolfe J, Vanderwalker ML, Nolop KB, Harrison JE: Mometasone furoate: efficacy and safety in moderate asthma compared with beclomethasone dipropionate. Ann Allergy Asthma Immunol 2001, 86(2):203-210.

14. Nayak AS, Banov C, Corren J, Feinstein BK, Floreani A, Friedman BF, Goldsobel A, Gottschlich GM, Hannaway PJ, Lampl KL, Lapidus RJ, Lawrence M, Lumry W, Munk Z, Pearlman D, Scardella AT, Schenkel EJ, 
Segal AT, Segall N, Silverman B, Shneyer L, Nolop KB, Harrison JE: Oncedaily mometasone furoate dry powder inhaler in the treatment of patients with persistent asthma. Ann Allergy Asthma Immunol 2000, 84(4):417-424.

15. Berger WE, Ford LB, Mahr T, Nathan RA, Crim C, Edwards L, Wightman DS, Lincourt WR, Rickard K: Efficacy and safety of fluticasone propionate 250 microg administered once daily in patients with persistent asthma treated with or without inhaled corticosteroids. Ann Allergy Asthma Immunol 2002, 89(4):393-399.

16. Chuchalin A, Jacques L, Frith L: Salmeterol/fluticasone propionate via Diskus once daily versus fluticasone propionate twice daily in patients with mild asthma not previously receiving maintenance corticosteroids. Clin Drug Investig 2008, 28(3):169-181.

17. Nelson HS, Busse WW, deBoisblanc BP, Berger WE, Noonan MJ, Webb DR, Wolford JP, Mahajan PS, Hamedani AG, Shah T, Harding SM: Fluticasone propionate powder: oral corticosteroid-sparing effect and improved lung function and quality of life in patients with severe chronic asthma. J Allergy Clin Immunol 1999, 103(2 Pt 1):267-275.

18. Chowdhury BA, Dal Pan G: The FDA and safe use of long-acting betaagonists in the treatment of asthma. N Engl J Med 2010, 362(13):1169-1171.

19. Walters EH, Gibson PG, Lasserson TJ, Walters JA: Long-acting beta2agonists for chronic asthma in adults and children where background therapy contains varied or no inhaled corticosteroid. Cochrane Database Syst Rev 2007, , 1: CD001385.

20. Cote C, Pearle JL, Sharafkhaneh A, Spangenthal S: Faster onset of action of formoterol versus salmeterol in patients with chronic obstructive pulmonary disease: a multicenter, randomized study. Pulm Pharmacol Ther 2009, 22(1):44-49.

21. van Noord JA, Smeets JJ, Raaijmakers JA, Bommer AM, Maesen FP: Salmeterol versus formoterol in patients with moderately severe asthma: onset and duration of action. Eur Respir J 1996, 9(8):1684-1688.

22. Murphy KR, Bender BG: Treatment of moderate to severe asthma: patient perspectives on combination inhaler therapy and implications for adherence. J Asthma Allergy 2009, 2:63-72.

23. Juniper EF, Bousquet J, Abetz L, Bateman ED: Identifying 'well-controlled' and 'not well-controlled' asthma using the Asthma Control Questionnaire. Respir Med 2006, 100(4):616-621.

24. Rachelefsky GS, Liao Y, Faruqi R: Impact of inhaled corticosteroid-induced oropharyngeal adverse events: results from a meta-analysis. Ann Allergy Asthma Immunol 2007, 98(3):225-238.

doi:10.1186/1710-1492-7-21

Cite this article as: Bernstein et al:. Efficacy and onset of action of mometasone furoate/formoterol and fluticasone propionate/salmeterol combination treatment in subjects with persistent asthma. Allergy, Asthma \& Clinical Immunology 2011 7:21.

\section{Submit your next manuscript to BioMed Central and take full advantage of:}

- Convenient online submission

- Thorough peer review

- No space constraints or color figure charges

- Immediate publication on acceptance

- Inclusion in PubMed, CAS, Scopus and Google Scholar

- Research which is freely available for redistribution

Submit your manuscript at www.biomedcentral.com/submit
Biomed Central 